\title{
Enumerating Galois Representations in Sage
}

\author{
Craig Citro ${ }^{12}$ and Alexandru Ghitza ${ }^{3}$ \\ 1 Google, Seattle WA \\ 2 Department of Mathematics, University of Washington, \\ Box 354350, Seattle WA 98195-4350, USA, \\ craigcitro@gmail.com \\ 3 Department of Mathematics and Statistics, University of Melbourne, \\ Parkville VIC 3010, Australia, \\ aghitza@alum.mit.edu
}

\begin{abstract}
We present an algorithm for enumerating all odd semisimple two-dimensional mod $p$ Galois representations unramified outside $p$. We also discuss the implementation of this algorithm in Sage and give a summary of the results we obtained ${ }^{4}$.
\end{abstract}

Key words: Galois representations, Sage, modular forms.

\section{Introduction}

A great deal of arithmetic questions have found natural interpretations (and often, answers) within the realm of Galois representations and modular forms: such applications include Diophantine equations, quadratic forms, or the study of combinatorial-arithmetic objects such as partitions. In this context, it is of interest to dispose of computational tools for working with modular forms and Galois representations.

In this note, we focus on two-dimensional Galois representations mod p, i.e. continuous group homomorphisms

$$
\rho: \operatorname{Gal}(\overline{\mathbb{Q}} / \mathbb{Q}) \longrightarrow \mathrm{GL}_{2}\left(\overline{\mathbb{F}}_{p}\right) .
$$

(More precisely, we consider such representations which are semisimple, unramified outside $p$, and odd. For the theoretical background, we refer the reader to Khare's survey [3] or to Edixhoven's paper [2].)

By Serre's conjecture, now a theorem of Khare-Wintenberger (see [4], [5]), these representations are closely related to modular forms $(\bmod p)$ of level 1 which are eigenvectors for all the Hecke operators. If $f$ is such a form, of weight $k$ and eigenvalues $\left(a_{\ell}\right)$, then for all primes $\ell \neq p$ we have

$$
\operatorname{charpoly}\left(\rho\left(\operatorname{Frob}_{\ell}\right)\right)=X^{2}-a_{\ell} X+\ell^{k-1},
$$

\footnotetext{
${ }^{4}$ The authors wish to thank Kevin Buzzard for providing several corrections and a significant improvement to Theorem 1, and the referees for suggesting improvements to the exposition.
} 
where Frob $\ell$ is a Frobenius element at $\ell$ inside $\operatorname{Gal}(\overline{\mathbb{Q}} / \mathbb{Q})$.

The (Hecke) eigensystem corresponding to a mod $p$ eigenform $f$ is the sequence $\left(a_{\ell}\right)$ of eigenvalues indexed by all primes $\ell \neq p$. The $i$-th twist of $\left(a_{\ell}\right)$ is by definition the eigensystem $\left(\ell^{i} a_{\ell}\right)$. We write $\left[a_{\ell}\right]$ for a finite truncation of $\left(a_{\ell}\right)$, where the cutoff point will be clear from the context.

Inspired by a remark of Khare ${ }^{5}$, we have set out to enumerate all odd semisimple $\bmod p$ representations which are unramified outside $p$. This corresponds to enumerating all the Hecke eigensystems which occur in spaces of level 1 modular forms $\bmod p$.

\section{Description of the Algorithm}

The starting point is a classical result in the theory of modular forms $\bmod p$ (see Theorem 3.4 in [2]): every Hecke eigensystem occurs, up to twist, in weights less than or equal to $p+1$. Therefore it suffices to generate the spaces $M_{k}$ for weights $4 \leq k \leq p+1$ and find all the eigenforms in them, which will produce all the Hecke eigensystems up to twist. This list may however contain duplicates; we investigate this question in detail in [1], where we prove

\section{Theorem 1.}

(a) Let $f_{1}$ and $f_{2}$ be eigenforms of weights $k_{1}, k_{2} \leq p+1$. If $f_{1}$ and $f_{2}$ have the same eigensystem up to twist, then $k_{1}+k_{2}=p+1$ or $k_{1}+k_{2}=p+3$.

(b) Let $f_{1}$ and $f_{2}$ be eigenforms of weights related in one of the ways described in (a). If $f_{1}$ and $f_{2}$ do not have the same eigensystem up to twist, then this is detected by a prime $\ell \neq p$ satisfying $\ell \leq(p+1) / 6$.

In the process of proving Theorem 1, we obtained the following lower bound, which improves the best known lower bound (due to Serre, see Sect. 8 in [3]) by a factor of two:

Theorem 2. Let $p>19$ be prime. The number of odd semisimple 2-dimensional Galois representations mod $p$ which are unramified outside $p$ is bounded below by $p(p-1) / 2$.

\section{Algorithm: Enumerate Galois representations $\bmod p$ up to twist}

1. For $4 \leq k \leq p+1$ :

(a) Compute a basis for the space $M_{k}$.

(b) Decompose the space into Hecke eigenspaces.

(c) For each eigenform, compute the eigenvalue $a_{\ell}$ of $T_{\ell}$ for primes $\ell$ up to the bound from Theorem 1 . Store $\left(k,\left[a_{\ell}\right]\right)$.

\footnotetext{
${ }^{5}$ From Sect. 8 of [3]: "[...] there are only finitely many semisimple 2-dimensional $\bmod p$ representations of $\operatorname{Gal}(\overline{\mathbb{Q}} / \mathbb{Q})$ of bounded (prime-to- $p$ Artin) conductor. It will be of interest to get quantitative refinements of this."
} 
2. Remove duplicates: given $\left(k_{1},\left[a_{\ell}\right]\right)$ and $\left(k_{2},\left[b_{\ell}\right]\right)$ such that $k_{1}+k_{2}=p+1$ or $p+3$, check whether $\left[b_{\ell}\right]$ is a twist of $\left[a_{\ell}\right]$.

This creates the list of equivalence classes (up to twist) of Hecke eigensystems $\bmod p$. It is now straightforward to apply the twist operation to each list element and generate the list of all Hecke eigensystems.

\section{Sage Implementation and Results}

Our task requires computing the action of Hecke operators on spaces of modular forms of high weight. Sage [8] offers several implementations of these spaces for arbitrary levels. We have initially used modular symbols over finite fields for generating the lists of eigenforms, but this method becomes quite slow as the weight increases. Restricting to level 1 allows us to take advantage of a much faster way of working with these spaces: the Victor Miller basis (see Sect. 2.3 in [7] for the properties and the algorithm Sage uses to compute this basis).

We then use one Hecke operator $T_{\ell}$ at a time to decompose the space $M_{k}$ into eigenspaces. This requires (at most) the first $k / 12$ primes $\ell$ (see the appendix of $[6])$.

We have run the Sage implementation of our algorithm for all primes up to 211 (see Table 1). Apart from keeping track of the number of equivalence classes of eigensystems and the total number of eigensystems, we save the list of equivalence classes; given this it is very easy to take twists and generate the entire list.

\begin{tabular}{r|r||r|r||r|r||r|r||r|r|r|r}
$p$ & number & $p$ & number & $p$ & number & $p$ & number & $p$ & number & $p$ & number \\
\hline 2 & 1 & 23 & 264 & 59 & 4234 & 97 & 19200 & 137 & 53992 & 179 & 119705 \\
3 & 1 & 29 & 532 & 61 & 4800 & 101 & 21600 & 139 & 55752 & 181 & 124020 \\
5 & 4 & 31 & 630 & 67 & 6237 & 103 & 22797 & 149 & 69264 & 191 & 145445 \\
7 & 9 & 37 & 1044 & 71 & 7420 & 107 & 25546 & 151 & 71700 & 193 & 150144 \\
11 & 35 & 41 & 1480 & 73 & 8136 & 109 & 27216 & 157 & 80340 & 197 & 160132 \\
13 & 48 & 43 & 1701 & 79 & 10257 & 113 & 30240 & 163 & 90477 & 199 & 164637 \\
17 & 112 & 47 & 2185 & 83 & 12054 & 127 & 42903 & 167 & 97276 & 211 & 196560 \\
19 & 153 & 53 & 3172 & 89 & 14784 & 131 & 46735 & 173 & 108016 & &
\end{tabular}

Table 1. Number of Galois representations $\bmod p$

Khare guesses in [3] that the number of Galois representations of the type we are considering should be asymptotic to $p^{3} / 48$. There are two phenomena that can contribute to the actual number being smaller than the guess: (i) the existence of "companion forms", which in our context appear as duplicate equivalence classes of eigenforms; (ii) the failure of "multiplicity one" for Hecke eigenvalues mod $p$, which results in some spaces $M_{k}$ not contributing their dimension's worth of eigenforms. In the range of our computations, the actual number of representations stays very close to the best known upper bound ${ }^{6}$, suggesting that the two phenomena are indeed quite rare. We expect this trend to be confirmed by further computations.

\footnotetext{
${ }^{6}$ For instance, for $p=211$ the quotient between the actual number (196560) and the upper bound (196665) is about 0.9995 .
} 


\section{References}

1. Citro, C., Ghitza, A.: Computing level 1 Hecke eigensystems $(\bmod p)$, preprint

2. Edixhoven, B.: The weight in Serre's conjectures on modular forms. Invent. Math. 109(3), 563-594 (1992)

3. Khare, C.: Modularity of Galois representations and motives with good reduction properties. J. Ramanujan Math. Soc. 22(1), 75-100 (2007)

4. Khare, C., Wintenberger, J.P.: Serre's modularity conjecture. I. Invent. Math. 178(3), 485-504 (2009), http://dx.doi.org/10.1007/s00222-009-0205-7

5. Khare, C., Wintenberger, J.P.: Serre's modularity conjecture. II. Invent. Math. 178(3), 505-586 (2009), http://dx.doi.org/10.1007/s00222-009-0206-6

6. Lario, J.C., Schoof, R.: Some computations with Hecke rings and deformation rings. Experiment. Math. 11(2), 303-311 (2002), http://projecteuclid.org/getRecord? id=euclid.em/1062621223, with an appendix by Amod Agashe and William Stein

7. Stein, W.: Modular forms, a computational approach, Graduate Studies in Mathematics, vol. 79. American Mathematical Society, Providence, RI (2007), with an appendix by Paul E. Gunnells

8. Stein, W., et al.: Sage Mathematics Software (Version 4.4.1). The Sage Development Team (2010), available from http://www.sagemath.org 